\title{
Hotéis e Operadoras de Viagens e Turismo: percepções distintas para um mesmo serviço
}

\section{Hotels and Tourism Operators: different perceptions for a same service}

\author{
Anderson Soares Lopes (LOPES, A. S.) ${ }^{*} \mathrm{e}$ \\ Jeferson Luís Mola (MOLA, J. L.) ${ }^{* *}$
}

RESUMO - Seriam idênticas as percepções dos gestores de hotéis e operadoras de turismo, no que diz respeito ao serviço prestado aos turistas de negócios? A partir de estudo em que se procurava entender como as demandas dos hóspedes organizacionais eram atendidas pelos meios de hospedagem e pelos intermediários desse processo, no presente estudo se avalia as dicotomias entre a opinião daqueles que Buhalis (2000) dá a entender como os dois principais stakeholders na prestação de serviços de turismo. Como método, na investigação se adota a pesquisa qualitativa e se usa como instrumento de coleta um questionário baseado na escala de diferenciação semântica (ou de Osgood), que foi aplicado a dois gestores de hotéis e à representante de uma operadora de viagens da cidade de São Paulo. No seu desenvolvimento, se compara e se discute a evolução do setor de turismo e hospitalidade no Brasil, contando para isso tanto com publicações nacionais quanto material internacional. $\mathrm{Na}$ análise dos dados se leva à consideração de que as percepções dos gestores de hotéis e operadoras são muito particulares - por vezes, díspares -, quando se pensa em prestação de serviços. Entendese que a prestação de serviços de qualidade seja uma forma saudável de competição, porém mesmo que a visão desses stakeholders seja semelhante em alguns aspectos, os resultados da pesquisa indicaram que o aumento de receita que a prestação de serviços poderia proporcionar a um meio de hospedagem transforma a satisfação do cliente de objetivo principal do prestador a uma forma relevante de gerar e/ou aproveitar boas oportunidades de negócio.

Palavras-chave: Hotéis; Operadoras de Viagens e Turismo; Hospitalidade; Serviços.

ABSTRACT - Would be perceptions of hotels and tour operators identical, as regards to the services provided to business tourists? From a paper which aimed to understand how the tourists demands would have been dealt by means of hosting and intermediaries in this process, the present study evaluates the dichotomies between the opinion of those that Buhalis (2000) considers the two main stakeholders in the provision of Tourism

\footnotetext{
Formação: Graduação em Turismo pela Associação Paraibana de Ensino Renovado (ASPER), Especialização em Gestão Hoteleira pela Castelli Escola Superior de Hotelaria e Mestrado em Hospitalidade pela Universidade Anhembi Morumbi. Membro dos grupos de pesquisa, Hospitalidade: Serviços e Organizações e CIDSGAM - Cidade Sustentável e Gestão Ambiental, também atuando como Colaborador da Meliá Hotels International. Endereço físico para correspondência: Rua Dr. Plínio Barreto, 389 (ap. 409) - Bela Vista. CEP: 01313-020 - São Paulo/SP - Brasil. E-mail: aslturjp@ yahoo.com.br

** Formação: Graduação em Comunicação Social - Publicidade e Propaganda pela Escola de Comunicações e Artes da Universidade de São Paulo (ECA/USP), Especialização MBA em Marketing pela Fundação Instituto de Administração da Universidade de São Paulo (FIA/USP) e em Administração Mercadológica pela Escola Superior de Propaganda e Marketing (ESPM/SP), Mestrado em Hospitalidade pela Universidade Anhembi Morumbi. Atividade profissional: Coordenador acadêmico e docente nos cursos de Marketing da Universidade Anhembi Morumbi. Endereço físico para correspondência: Rua Capote Valente, 411 (ap.) 603. CEP 05409-000, São Paulo/SP - Brasil. E-mail: j.mola@ hotmail.com
} 
services. The method adopted was the qualitative research which used as instrument for data collecting a questionnaire based on semantic differentiation scale (or Osgood scale), which was applied to two hotel managers and to a travel operator representative from São Paulo. In its development, the article compares and discusses the evolution of the Tourism and hospitality sector in Brazil, using for it both national and international publications. The analysis of the data leads to the consideration that the perceptions of hotels managers and tour operators are quite specific - sometimes disparate - when they think in provision of service. It is understood that the provision of quality services is a healthy form of competition and, although stakeholders' opinion is similar in some ways. The results indicate that the increase of revenue obtained through services may turn customer satisfaction from the primary purpose of a means of hosting to an interesting opportunity of doing business.

Key words: Hotels; Tourism Operators; Hospitality; Services. 


\section{INTRODUÇÃO}

No presente estudo se busca tecer considerações a respeito da relação entre hotéis e operadoras de turismo. Também se avalia a prestação de serviços no ambiente dos hotéis, sobretudo ao analisar e investigar de que forma os gestores das operadoras de turismo atuam e qual seu papel como um dos canais de venda fundamentais para o setor de hospedagem.

Para a Organização Mundial do Turismo apud Buhalis (2000) ${ }^{1}$, os canais de distribuição no turismo são os agentes de viagens, os operadores de viagens e turismo, os sistemas de reservas e outros especialistas. Buhalis (2000), no entanto, destaca que os estudos realizados nas vertentes do turismo sempre contam com a presença de dois intermediários, mais bem exemplificados na forma de operadores e agências de viagens.

Este artigo é consequência de uma pesquisa anterior, elaborada para o X Seminário da Associação Nacional de Pesquisa e Pós-graduação em Turismo ANPTUR, em que se buscava identificar - sob a ótica dos gestores de hotéis, operadoras de turismo e dos próprios hóspedes - quais seriam os serviços considerados essenciais para os turistas de negócio na cidade de São Paulo (LOPES; MOLA, 2013).

Diante dos resultados obtidos com aquele trabalho, observou-se que, no atual cenário, algumas indagações poderiam ser mais bem esclarecidas e investigadas por meio de uma nova pesquisa. Neste estudo se busca, portanto, respostas para tais indagações e o esclarecimento de aspectos levantados em pesquisa anterior e, nesse contexto, considera-se que o processo de pesquisa e aprendizagem tende a ser uma atividade constante, na qual o pesquisador busca integrar conhecimentos advindos dos múltiplos campos das ciências para, desta forma, utilizá-los como ferramenta à compreensão do comportamento humano.

Verificam-se, assim, as palavras de Panosso Netto e Calciolari (2010, p. 669), para quem "o desenvolvimento de qualquer área científica está diretamente relacionado, dentre outros fatores, com a quantidade e, principalmente, com a qualidade da pesquisa feita e publicada em tal área". Espera-se, portanto, que este trabalho agregue conhecimento ao setor de turismo e hospitalidade.

\footnotetext{
${ }^{1}$ WTO, 1975, Distribution channels, World Tourism Organization, Madrid.
} 
$\mathrm{Na}$ primeira pesquisa empreendida anterior a esse artigo, de natureza exploratória, se buscou investigar e compreender o fenômeno da hospedagem e se utilizou de livros e periódicos nacionais e internacionais, bem como de publicações dispostas em revistas especializadas na área de turismo e hotelaria (BRASIL, 2015; GUERRA, 2001; SENAC, 2013). Buscava-se, com isso, o debate a respeito do saber localizado nos limites mercadológicos, com o conhecimento produzido e disseminado nos limites das Instituições de Ensino Superior - IES. Os pesquisadores autores também procuraram integrar o conhecimento disponibilizado nos limites territoriais do Brasil com as publicações efetuadas em outros países.

Posteriormente, adotou-se uma pesquisa de campo, cuja amostragem se constituiu de dois gestores de hotéis e de uma representante de operadora de viagens e turismo. Os gestores estavam atuando em empreendimentos localizados nos limites territoriais da cidade de São Paulo e, assim como a representante da operadora, responderam a um questionário em que se buscava comparar a relevância de determinados serviços oferecidos aos turistas de negócios na cidade de São Paulo.

No questionário se tomou por base uma escala de diferencial semântico e apresentava sempre duas opções de serviços: uma associada à oferta desse serviço no próprio estabelecimento e outra referente à localização de prestadores do serviço nas proximidades do meio de hospedagem. A relevância de tais serviços era determinada por meio de uma escala com valores de zero a três. Cada uma das opções era apresentada de um dos lados dessa escala e o respondente determinava, segundo sua percepção, a relevância de um serviço em comparação ao outro. Isto permitia que, quanto mais relevante fosse considerado um serviço (em detrimento do outro), maior seria a pontuação atribuída a ele. No centro dessa escala, encontrava-se uma coluna de valor zero que podia ser escolhida pelo respondente e indicaria, nesse caso, que - para ele - as alternativas em questão teriam grau de importância similar.

Com tal pesquisa, buscava-se localizar que serviços seriam essenciais para um turista de negócios na cidade de São Paulo a partir da ótica dos gestores de hotéis e das operadoras de turismo e o modelo de questionário proposto tinha por objetivo simplificar não apenas o processo de condução de perguntas e respostas, mas também o tratamento dos dados e a análise de resultados. 
Como adendo ao processo, vale ressaltar que os dois gestores pesquisados responderam à pesquisa pessoalmente, sem intervenção dos pesquisadores no preenchimento dos questionários, e que a representante da operadora de viagens o fez por e-mail, devido às limitações de tempo e distância para um contato pessoal.

Foi possível detectar que o gestor do primeiro hotel - localizado no bairro da Chácara Santo Antonio e próximo às vias de acesso rápido Marginal Pinheiros e Avenida Santo Amaro - entendeu que o empreendimento pelo qual era responsável visava a atender ao público de negócios, enquanto o gestor do segundo hotel estabelecido no bairro da Vila Olímpia, região que em que se mesclavam escritórios e bares e ficava próximo à Avenida dos Bandeirantes, um corredor de trânsito importante da cidade - percebeu que a proposta da bandeira que representava estava na prestação de serviços associada à versatilidade, ou seja, ao fato de que os colaboradores do hotel estariam preocupados em prestar serviços de forma personalizada, adequando-se às necessidades de cada hóspede.

A terceira entrevistada estava atuando como representante do Grupo Alatur que, embora atuasse tanto nos segmentos de viagens de lazer e férias ou de viagens de incentivos, estava sendo uma das empresas líderes no segmento TMC - Travel Management Companies, no Brasil. Dentre os serviços disponibilizados pela Alatur JTB encontravam-se as reservas de viagens aéreas, reservas de hotéis, locação de veículos, passagens rodoviárias e ferroviárias e orientação de documentação para viagens, em atendimento disponível 24 horas (ALATUR JTB, 2015).

O grupo estava possuindo parcerias estratégicas com empresas em distintas localidades, em diversos setores do turismo, e seu alcance de atuação era global. Podem ser destacadas, dentre essas parcerias (ALATUR JTB, 2015):

- HRG Worldwide - Hogg Robinson Group, empresa especializada na gestão de viagens corporativas;

- MCI Group, atuante no gerenciamento de associações, comunicação e eventos corporativos;

- Rede de agências de alto luxo Virtuoso;

- Redes de hotéis Four Seasons e The Ritz-Carlton.

Dentre os fatores que concediam um status diferenciado às organizações acima descritas, podem-se citar a liderança de mercado que possuíam em suas áreas de atuação 
e o alto grau de qualidade que buscavam oferecer na prestação de seus serviços. Além dessas, o Grupo Alatur estava associado a outras organizações de elevado prestígio no contexto global (ALATUR JTB, 2015), como:

- Meeting Professional International - MPI, associação global para eventos profissionais;

- Association Corporate Travel Executives - ACTE Global e Associação Latino Americana de Gestores de Viagens Corporativas - ALAGEV, que representam os gestores desse tipo de viagem.

Em meio a tais considerações, entendeu-se como problema desta pesquisa a necessidade de verificar se os gestores dos hotéis da cidade de cidade de São Paulo e os das operadoras de viagens e turismo possuíam o mesmo entendimento quanto aos serviços disponibilizados para os hóspedes do setor corporativo.

A priori, nas hipóteses se buscavam investigar conceitos associados às palavraschaves da pesquisa: (1) Que os gestores dos hotéis e operadoras de viagens e turismo possuíam o mesmo entendimento a respeito dos serviços, ditos essenciais, que eram disponibilizados aos hóspedes e (2) Que a relação entre hotéis e operadoras de viagens e turismo acontecia de forma amistosa.

No artigo se tem, por objetivo geral, o intuito de analisar a relação entre os gestores das operadoras de viagens e turismo e hotéis na cidade de São Paulo, e se buscou verificar se a relação que se estabelecia entre prestadores de serviços e canais de venda acontecia de forma harmoniosa, constituindo-se em atividade benéfica para ambas as partes. Considera-se que a relevância do estudo está vinculada à contribuição teórica que permitirá analisar os segmentos, setores e organizações que - por sua abrangência ou características - carecem de maior volume de informações para o seu desenvolvimento.

\section{SEGMENTAÇÃO DE MERCADO NOS HOTÉIS DA CIDADE DE SÃo PAULO}

No decorrer da evolução do turismo, constata-se que este enquanto atividade tem se tornado mais complexo, exigindo elevado fluxo de informações entre os distintos stakeholders desta atividade (SANTOS; SANTOS, 2014), assim incorporando em seu 
campo de estudo não apenas os meios de hospedagem tradicionais, mas também distintas organizações de/para suporte aos negócios.

Além de colaborarem com o desenvolvimento de um destino turístico, os gestores destas organizações demonstram, por sua abrangência de atuação e enfoque, compreender os efeitos positivos que sua atividade proporciona ao setor.

Tal fato se traduz na incorporação de diferentes interessados, ou seja, organizações que prestam serviços com finalidades distintas e que, na estrutura produtiva do setor turístico, podem ser classificadas como os "meios de hospedagem, operadoras de turismo, empresas do setor de alimentação, agentes de viagens, empresas de entretenimento, organizações do setor de transporte e a comunidade residente no polo turístico" (NASCIMENTO; LOPES, 2012, p. 8).

Um dos fatores em que se constata o crescimento do setor de turismo no Brasil é o aumento do fluxo internacional ao País no decorrer das últimas duas décadas. Para Panosso Netto e Calciolari (2010, p. 683) isto ocorre "pela compreensão do que o fenômeno turístico tem representado para o país como fonte de riquezas e de desenvolvimento humano e social". Estudos de caráter acadêmico e governamental, como o levantamento efetuado pelo Departamento de Polícia Federal em conjunto com o Ministério do Turismo, em seu Anuário Estatístico do Turismo (BRASIL, 2013), corroboram essa tese, como pode ser visto na Tabela 1 - Chegadas de turistas ao Brasil, segundo os anos 1970-2012.

TABELA 1 - CHEGADAS DE TURISTAS AO BRASIL, SEGUNDO OS ANOS 1970-2012

\begin{tabular}{|c|c|c|c|c|c|c|c|}
\hline \multicolumn{1}{|c|}{ CHEGADAS DE TURISTAS } \\
\hline $\mathbf{1 9 7 0}$ & Total & Ano & Total & Ano & Total & Ano & Total \\
\hline $\mathbf{1 9 7 1}$ & 289.900 & $\mathbf{1 9 8 2}$ & 1.146 .681 & $\mathbf{1 9 9 4}$ & 1.853 .301 & $\mathbf{2 0 0 6}$ & 5.017 .251 \\
$\mathbf{1 9 7 2}$ & 342.961 & $\mathbf{1 9 8 4}$ & 1.595 .726 & $\mathbf{1 9 9 6}$ & 2.665 .508 & $\mathbf{2 0 0 8}$ & 5.050 .099 \\
$\mathbf{1 9 7 3}$ & 399.127 & $\mathbf{1 9 8 5}$ & 1.735 .982 & $\mathbf{1 9 9 7}$ & 2.849 .750 & $\mathbf{2 0 0 9}$ & 4.802 .217 \\
$\mathbf{1 9 7 4}$ & 480.267 & $\mathbf{1 9 8 6}$ & 1.934 .091 & $\mathbf{1 9 9 8}$ & 4.818 .084 & $\mathbf{2 0 1 0}$ & 5.161 .379 \\
$\mathbf{1 9 7 5}$ & 517.967 & $\mathbf{1 9 8 7}$ & 1.929 .053 & $\mathbf{1 9 9 9}$ & 5.107 .169 & $\mathbf{2 0 1 1}$ & 5.433 .354 \\
\hline 1976 & 555.967 & $\mathbf{1 9 8 8}$ & 1.742 .939 & $\mathbf{2 0 0 0}$ & 5.313 .463 & $\mathbf{2 0 1 2}$ & 5.676 .843 \\
\hline
\end{tabular}




\begin{tabular}{|ll|l|l|ll|l|} 
& & & & & & Continuação... \\
$\mathbf{1 9 7 7}$ & 634.595 & $\mathbf{1 9 8 9}$ & 1.402 .897 & $\mathbf{2 0 0 1}$ & 4.772 .575 & \\
$\mathbf{1 9 7 8}$ & 784.316 & $\mathbf{1 9 9 0}$ & 1.091 .067 & $\mathbf{2 0 0 2}$ & 3.784 .898 & \\
\hline $\mathbf{1 9 7 9}$ & 1.081 .799 & $\mathbf{1 9 9 1}$ & 1.228 .178 & $\mathbf{2 0 0 3}$ & 4.132 .847 & \\
\hline $\mathbf{1 9 8 0}$ & 1.625 .422 & $\mathbf{1 9 9 2}$ & 1.692 .078 & $\mathbf{2 0 0 4}$ & 4.793 .703 & \\
\hline $\mathbf{1 9 8}$ & 1.357 .879 & $\mathbf{1 9 9 3}$ & 1.641 .138 & $\mathbf{2 0 0 5}$ & 5.358 .170 & \\
\hline
\end{tabular}

Fonte: Departamento de Polícia Federal e Ministério do Turismo - Anuário Estatístico do Turismo (Brasil, 2013).

A análise dessa tabela permite verificar o aumento do fluxo turístico internacional em território nacional ao longo dos anos: segundo os dados aí expostos, o número de turistas estrangeiros em visita ao Brasil em 2012 foi 22 vezes maior do que em 1970. Constata-se um aumento acentuado nessa atividade a partir de 1998, possivelmente por terem sido criadas, nessa época, políticas de turismo e formas de promoção do País adequadas aos padrões internacionais.

Uma avaliação crítica do setor de turismo e hospitalidade no Brasil indica, no entanto, que o país se encontra em uma situação inferior a suas verdadeiras possibilidades - principalmente quando se compara a realidade nacional ao cenário de qualificação e prestação de serviços vivenciado nos países considerados de primeiro mundo. Ainda assim, o aumento do fluxo turístico no País é consequência de uma ação sinérgica entre o governo e a iniciativa privada e tem por objetivo capacitar a força de trabalho visando ao atendimento das necessidades do turista.

Por essa razão, pode-se afirmar que os serviços em turismo no Brasil contemporâneo apresentam - ainda que tal afirmação apresente ressalvas - um grau crescente de qualificação e que os gestores das organizações atuantes no setor parecem estar mais atentos às expectativas e aos comportamentos do consumidor, procurando satisfazê-lo para, com isto, construir relacionamento, gerar fidelização e - sem dúvida fortalecer a própria posição de mercado.

No que se refere a tais organizações, a manutenção de um posicionamento adequado e o melhor aproveitamento das oportunidades mercadológicas são uma necessidade e, para Castells (2000, p. 9), esta atividade pode ser resumida da seguinte forma: 
In the last two decades of the twentieth century a related set of social transformations has taken place around the world. While cultures, institutions, and historical trajectories introduce a great deal of diversity in the actual manifestations of each one of these transformations, it can be shown that, overall, the vast majority of societies are affected in a fundamental way by these transformations ${ }^{2}$.

Estas transformações geram desejos distintos nos consumidores: se o mercado busca ofertar diferenciais, então um cliente tem o direito de exigir - como característica do valor desse diferencial - a personalização de um produto ou serviço e a atenção individualizada a seus interesses e/ou necessidades. Sob essa ótica, o ato de segmentar o mercado e oferecer produtos e serviços exclusivos a grupos distintos - porém semelhantes - passa a ser uma atividade intrínseca ao sucesso ou ao insucesso de um negócio relacionado a esse setor.

Ao caminhar em paralelo ao setor turístico, o setor de hospedagem evoluiu e, por consequência, acabou acompanhando as mudanças e tendências surgidas no mercado global, tanto nos aspectos tecnológicos quanto socioeconômicos ou político-legais. Na Tabela 2 - Projeção da oferta e demanda do setor hoteleiro na cidade de São Paulo se apresenta um exemplo da segmentação do mercado e nela se faz uma projeção da demanda e da oferta nesse setor para o ano de 2015.

TABELA 2 - PROJEÇÃO DA OFERTA E DEMANDA NO SETOR HOTELEIRO NA CIDADE DE SÃO PAULO

\begin{tabular}{|c|c|c|c|c|c|c|c|}
\hline \multicolumn{5}{|c|}{ INFORMAÇÕES ANO BASE } & \multicolumn{3}{|c|}{ PROJEÇÃO PARA 2015} \\
\hline & $\begin{array}{l}\text { Oferta } \\
\text { Atual } \\
\left.\text { (UH's }^{3}\right)\end{array}$ & $\begin{array}{l}\text { Demanda } \\
\text { Atual } \\
\text { (UH's) }\end{array}$ & $\begin{array}{l}\text { T. O. } \\
2011\end{array}$ & $\begin{array}{l}\text { C. M. } \\
\text { D. } \\
\text { A. }{ }^{5}\end{array}$ & $\begin{array}{l}\text { Oferta } \\
\text { Adicional } \\
\text { (UH's) }\end{array}$ & $\begin{array}{l}\text { Demanda } \\
\text { Adicional } \\
\text { (UH's) }\end{array}$ & $\begin{array}{l}\text { T. O. } \\
2015\end{array}$ \\
\hline TOTAL & 35.869 & 24.374 & $68 \%$ & $3.9 \%$ & 463 & 4.063 & $78 \%$ \\
\hline ECONÔMICO & 14.547 & 10.183 & $70 \%$ & $4.1 \%$ & 120 & 1.768 & $81 \%$ \\
\hline$\overline{\text { MIDSCALE }}$ & 16.582 & 11.110 & $67 \%$ & $4.3 \%$ & 343 & 2.058 & $78 \%$ \\
\hline UPSCALE & 4.740 & 3.081 & $65 \%$ & $2.0 \%$ & 0 & 237 & $70 \%$ \\
\hline
\end{tabular}

Fonte: Placar da Hotelaria (SENAC SÃO PAULO, 2013).

\footnotetext{
${ }^{2}$ Nas duas últimas décadas do século $\mathrm{XX}$, um conjunto relacionado de transformações sociais tem ocorrido em todo o mundo. Enquanto as culturas, instituições e trajetórias históricas introduziram uma grande quantidade de diversidade nas manifestações reais de cada uma dessas transformações, pode-se mostrar que, em geral, a grande maioria das sociedades são afetadas de uma maneira fundamental por essas transformações (tradução dos autores).

${ }^{3}$ Unidades Habitacionais.

${ }^{4}$ Taxa de Ocupação.

${ }^{5}$ Crescimento Médio da Demanda Acomodada (no ano).
} 
Pela análise dessa tabela, verifica-se que a oferta e a demanda por meios de hospedagem na cidade de São Paulo tende a crescer mesmo após um evento como a Copa do Mundo de Futebol da FIFA 2014. A cidade apresentando mercado hoteleiro favorável, com níveis de ocupação permitindo boas tarifas, margens operacionais satisfatórias e rentabilidade sobre o investimento adequada (SENAC, 2013). Por apresentar tais requisitos, a cidade de São Paulo foi escolhida como fonte de pesquisa neste artigo.

Os dados publicados no Placar da Hotelaria demonstram que o setor hoteleiro vivencia uma sistematização de conceitos e conhecimentos relacionados às áreas de marketing e administração e que o risco de superoferta de hotéis no período pós-Copa, considerável em cidades como Belo Horizonte, Brasília, Cuiabá, Manaus, Salvador e Recife no segmento midscale, em Porto Alegre nos segmentos econômico e midscale e no Rio de Janeiro no segmento midscale, merecendo atenção especial por parte dos empreendedores voltados a este setor (SENAC, 2013).

A atividade de segmentar os meios de hospedagem e classificá-los por tipos e categorias é efetuada, no Brasil, pelo Ministério do Turismo, que utiliza - como símbolo de identificação de um empreendimento - a figura da estrela (BRASIL, 2015). Verifica-se, desta forma, que também o governo busca sistematizar conceitos no setor de hospedagem, de modo a facilitar a escolha dos consumidores.

Sistematizar conceitos de diferentes áreas do conhecimento, investir na melhoria das instalações e inserir inovações tecnológicas nos meios de hospedagem com vistas à maior comodidade dos clientes e à adequação do espaço e dos serviços às suas necessidades/desejos, passaram a ser fator crítico para o sucesso no setor.

Em um mundo globalizado, as tendências são mais variadas - e, por vezes, efêmeras. Este processo vem se acelerando ao longo das últimas duas décadas e tem tornado mais desafiadora a análise do setor por parte de seus estudiosos. Um tipo de análise voltado à hospitalidade do turismo precisa estar ligado ao dia a dia dos negócios, mas não pode deixar de lado a conjuntura mundial. Otto (2013) apresenta a partir da relação que faz entre os estudos desenvolvidos pelas organizações Deloitte Consulting 
Services $^{6}$ e Travel + Leisure $^{7}$, uma série de tendências a que as empresas do setor deveriam atentar-se:

1 - Investir em agilidade, simplificando processos e diminuindo o custo de distribuição dos produtos e serviços de forma inteligente;

2 - Aperfeiçoar a experiência do consumidor, gerando fidelização;

3 - Inovar nas mídias sociais, ampliando a comunicação e criando novas formas de distribuição dos serviços;

4 - Adaptar-se às novas gerações, proporcionando diversos meios de interação do cliente com a empresa.

Otto (2013) destaca, ainda, a tendência da sustentabilidade, por meio da qual os gestores das empresas deveriam buscar oportunidades e formas de respeitar o meio ambiente - ainda que sob a lógica capitalista. Para a autora, isto tende a agregar valor ou um status diferenciado para a empresa frente à concorrência, e pode atrair maior número de clientes.

É fato, porém, que as empresas voltadas ao setor hoteleiro possuem valores essenciais, focados no relacionamento e na prestação de serviços: a hospitalidade se constitui na atividade central de um meio de hospedagem e, como tal, estabelece a alma de um empreendimento: o bem receber e bem atender as aspirações de hóspedes, visitantes, colaboradores e demais stakehoders da organização.

\section{A RELAÇÃO ENTRE OS HOTÉIS E AS OPERADORAS DE TURISMO}

Para as empresas atuantes no mercado de turismo global, a maximização dos índices operacionais e financeiros é um imperativo. Como no presente artigo buscou-se considerar a atuação dos hotéis e das operadoras de turismo, entende-se que nesta relação são primordiais os graus de influência de ambos os negócios no ambiente nacional e internacional.

\footnotetext{
6 Disponível em: <http://www2.deloitte.com/sa/en/pages/human-capital/articles/global-human-capitaltrends-2013.html>.

${ }^{7}$ Disponível em: <http://www.travelandleisure.com/slideshows/most-important-travel-trends-2014>.
} 
No bojo desta atividade, busca-se garantir para os hotéis ocupação e rentabilidade e, para as operadoras de turismo, a capacidade de ofertar produtos de hospedagem de acordo os desejos dos clientes. Desta maneira, hotéis e operadoras de turismo visam a demonstrar excelência na prestação de serviços ao consumidor e, a partir disso, garantir (ou, pelo menos, tentar garantir) a satisfação de todas as partes envolvidas no processo.

Nesse cenário, as operadoras de turismo possuem alguma vantagem: uma tendência natural no mercado de turismo são a atuação e a expansão das OTAs - Online Travel Agencies, que desenvolvem suas atividades em um ambiente ainda bastante "centralizado, com custos reduzidos, não dispondo de um atendimento presencial aos clientes, de uma equipe grande, entre outros" (ESTRUQUEL, 2013, p. 2). Ao facilitar a distribuição de um produto hoteleiro, uma OTA é, ao mesmo tempo, parceira e concorrente importante das agências tradicionais de viagens.

Percebe-se, por esse modelo, que existe um conflito de interesses na relação entre hotéis, agências de viagens online e tradicionais. Porém constata-se que - mesmo sem estudos prévios - os avanços tecnológicos oriundos dessa nova forma de comercialização acabam por tornar o produto hoteleiro mais acessível e, de alguma forma, contribuem para a expansão do mercado e para o aumento do número de consumidores. Nessa perspectiva verifica-se que a introdução de novas tecnologias relacionadas ao setor turístico vem a ser utilizadas para atender os turistas, agregar valor aos produtos e serviços, e por fim, criar novas oportunidades para as organizações e localidades (SANTOS; SANTOS, 2014).

Para a BUHALIS (2000, p. 114), "the position of the distribution sector is much stronger: trade intermediaries [...] have a far greater power to influence and to direct demand than their counterparts in other industries do" ${ }^{\prime 8}$. Neste contexto, compreende-se que hotéis e operadoras precisam trabalhar em conjunto e, no decorrer de suas atividades, deverão atuar em sintonia.

Para diminuir as divergências entre estes dois tipos de empresas, torna-se primordial o compartilhamento da informação - pelo uso de web sites, participação em redes sociais, envio de e-mails ou telefonemas. Buhalis (2000, p. 114) entende que,

\footnotetext{
8 “a posição do setor da distribuição é muito mais forte: o grupo de intermediários [...] tem um poder muito maior de influenciar e direcionar a demanda do que outras empresas em outros setores" (tradução dos autores).
} 
nessa esfera de negócios, "promotional element is still ignored, but the information provision function is highlighted" $"$.

Isto se deve pela atenção e pelo desejo dos gestores e colabores de minimizar falhas e interferências operacionais e/ou processuais, facilitando o processo de aquisição e venda da hospedagem para o consumidor. Divergências nessa relação tendem a fazer com que todas as partes percam energia.

Imagine-se o caso (real) do vice-presidente de uma companhia alemã que, ao chegar a um hotel por volta das 23 horas, descobre que a reserva não fora efetuada e que esse meio de hospedagem (o mais próximo do local da reunião que ele conduzirá a partir das 7 horas do dia seguinte) encontra-se lotado. Sem dúvida, o responsável pelo próprio meio de hospedagem tentará resolver o impasse, buscando outro hotel da mesma categoria (se possível, da mesma rede) para hospedar o turista. Mas as consequências negativas dessa falha poderão afetar profundamente a relação entre as partes e, possivelmente, tanto o meio de hospedagem quanto o canal de distribuição encontrarão dificuldades para alcançar seus objetivos se quiserem captar novos hóspedes dessa mesma empresa.

Este caso ilustra o fato - que também a literatura permite verificar - de que a troca constante de informações entre o meio de hospedagem e os seus canais de distribuição é fator intrínseco nesta relação. Como afirma Buhalis (2000, p. 114), os canais de distribuição no turismo "attract more attention by contemporary researchers and strategists" 10 pois a atividade que desempenham independe de fórmulas e, por isso mesmo, se constituem em tarefa das mais complexas.

Para demonstrar tamanha complexidade, considerem-se os períodos de sazonalidade do turismo, nos quais os hotéis também se encontram aparentemente expostos: um evento como a Fórmula 1, por exemplo, gera um número muito grande de deslocamentos durante uma semana - normalmente no mês de novembro -, mas tem levado os turistas que desembarcam em São Paulo a sofrerem, de forma constante, com a falta de leitos.

\footnotetext{
9 “elementos promocionais ainda são ignorados, mas a função de fornecimento de informações é destaque" (tradução dos autores).

10 “atrair mais a atenção de pesquisadores e estrategistas contemporâneos” (tradução dos autores).
} 
Em contrapartida, a constatação da vocação da cidade para o turismo de negócios, já que se observa segundo o Convention \& Visitors Bureau (2015) que 51,2\% dos turistas se deslocam para São Paulo por motivo predominante negócios, devido a este fato os meses de maior ocupação em 2014 foram Maio, Agosto e Novembro (CVB, 2015). Por isso, além de contar com equipamentos e serviços para fins turísticos de qualidade, a cidade de São Paulo voltada ao turista de negócios precisa dispor de outras ferramentas mercadológicas se quiser solucionar a questão da baixa ocupação. Isto, para Buhalis (2000, p. 116), tende a se tornar uma realidade, afinal:

Contemporary channels not only distribute tourism products, but also influence all the other elements of the marketing mix. For example, channels often determine the price by assessing real-time demand and available supply; manipulate and formulate tourism products by combining and tailoring products according to customers' needs and wishes; and finally facilitate promotion by targeting specific markets and establishing communication $^{11}$.

A combinação e a utilização das distintas ferramentas de marketing como meio de potencializar os canais de distribuição do setor turístico brasileiro tende a ultrapassar o ambiente interno das organizações e poderia ser um modo de influenciar fluxos turísticos em períodos de alta ou baixa demanda, contribuindo para a formulação de políticas de promoção e gestão mais específicas e eficientes.

Em artigo publicado na Revista Hotéis, Estruquel (2013) destaca cinco pontos relevantes na parceria entre agências de viagens, operadoras e meios de hospedagem: (1) definição do produto comercializado; (2) posicionamento no mercado; (3) política comercial definida; (4) cumprimento das políticas acordadas e, por fim, (5) cumprimento das metas comerciais estabelecidas e definidas entre as empresas.

Os responsáveis pelas organizações já entendem que uma boa estratégia de marketing é capaz de influenciar hábitos e costumes e que - como lembra Estruquel (2013, p. 1) - a evolução dos negócios dependerá da "relação transparente, harmoniosa e de confiança" entre as partes, gerando lucro para o empreendimento e satisfação para o cliente.

\footnotetext{
${ }^{11}$ Canais contemporâneos não só distribuem produtos turísticos, mas também influenciam todos os outros elementos do mix de marketing. Por exemplo, os canais muitas vezes determinam o preço, avaliando a demanda em tempo real e a oferta disponível, manipulam e formulam produtos turísticos por meio da combinação de produtos de acordo com as necessidades dos clientes e desejos e, finalmente, facilitam a promoção, visando mercados específicos e estabelecendo a comunicação (tradução dos autores).
} 


\section{ANÁLISE DOS DADOS}

Por meio de uma ferramenta de pesquisa baseada na escala de diferencial semântico, os representantes de dois hotéis estabelecidos na cidade de São Paulo e da operadora Alatur avaliaram uma série binária de 18 tipos de serviços oferecidos ao turista de negócios e os resultados dessa percepção são apresentados a seguir. Ao buscar as semelhanças e diferenças de opiniões desses dois stakeholders, na presente pesquisa se pretendeu compreender o cenário e, a partir dessa compreensão, propor um direcionamento às ações mercadológicas dos players do setor.

O tratamento dos dados permitiu a elaboração dos gráficos abaixo e a análise desses gráficos mostrou que as percepções dos gestores e da representante da operadora de viagens são, muitas vezes, diametralmente opostas. Ainda assim - ou talvez exatamente por isso - entende-se que tamanha divergência de percepções enriquece o debate e abre novas perspectivas ao estudo e ao desenvolvimento do setor de turismo no Brasil.

GRÁFICO 1 - PREFERÊNCIA DOS HOTÉIS E OPERADORA EM RELAÇÃO AO SERVIÇO DE ROOM SERVICE X INDICAÇÃO DE RESTAURANTES PRÓXIMOS AO MEIO DE HOSPEDAGEM

\begin{tabular}{|c|c|c|c|c|c|c|c|c|c|c|c|c|}
\hline \multicolumn{6}{|c|}{ Serviço de room service 24 horas } & & \multicolumn{6}{|c|}{ Indicação de restaurantes próximos } \\
\hline 3,0 & 2,5 & 2,0 & 1,5 & 1,0 & 0,5 & 0,0 & \multirow[t]{3}{*}{, } & \multirow[t]{3}{*}{1,0} & \multirow[t]{3}{*}{1,5} & \multirow[t]{3}{*}{2,0} & \multirow[t]{3}{*}{2,5} & \multirow[t]{3}{*}{3,0} \\
\hline \multicolumn{6}{|c|}{ OPERADORA } & & & & & & & \\
\hline & & & OTÉ & & & & & & & & & \\
\hline
\end{tabular}

Fonte: Gráfico elaborado pelos autores (2015).

O Gráfico 1 revela que tanto a gestora da operadora quanto dos hotéis concederam grande importância ao serviço de room service. A menor relevância que a representante da operadora pesquisada deu a esse serviço, no entanto, denotou os papéis distintos de cada empresa: distante dos interesses exclusivos do hotel, para a operadora o room service representaria, em si, uma praticidade ao hóspede, enquanto para o hotel, a prestação desse serviço poderia estar atrelada à comercialização de mais produtos e a uma fonte adicional de vendas. 
GRÁFICO 2 - PREFERÊNCIA DOS HOTÉIS E OPERADORA EM RELAÇÃO AO SERVIÇO DE BUSINESS CENTER X INTERNET WI FI CORTESIA

\begin{tabular}{|c|c|c|c|c|c|c|c|c|c|c|c|c|}
\hline \multicolumn{6}{|c|}{ Serviço de business Center } & & \multicolumn{6}{|c|}{ Internet wi fi cortesia } \\
\hline 3,0 & 2,5 & 2,0 & 1,5 & 1,0 & 0,5 & 0,0 & 0,5 & 1,0 & 1,5 & 2,0 & 2,5 & 3,0 \\
\hline & & & & & & & \multicolumn{6}{|c|}{ OPERADORA } \\
\hline & & & & & & & \multicolumn{6}{|c|}{ HOTEÍS } \\
\hline
\end{tabular}

Fonte: Gráfico elaborado pelos autores (2015).

O consenso obtido no Gráfico 2 mostra claramente que o serviço de Internet wi fi cortesia é importante para o turista de negócios, mas implicitamente também revela que, ao investir nessa forma de comunicação - na qual um hóspede usa computador próprio, e pode se conectar à rede em qualquer ambiente ou em qualquer horário - os hotéis geram economia: o investimento em equipamentos, pessoal ou manutenção, se não podem ser eliminados, com certeza poderão ser reduzidos.

GRÁFICO 3 - PREFERÊNCIA DOS HOTÉIS E OPERADORA EM RELAÇÃO AO SERVIÇO DE ACADEMIA E LAZER X PROXIMIDADE A CLUBES

\begin{tabular}{|c|c|c|c|c|c|c|c|c|c|c|c|c|}
\hline \multicolumn{6}{|c|}{ Serviços de academia e lazer } & & \multicolumn{6}{|c|}{ Localização próxima a clubes } \\
\hline 3,0 & 2,5 & 2,0 & 1,5 & 1,0 & 0,5 & 0,0 & 0,5 & 1,0 & 1,5 & 2,0 & 2,5 & 3,0 \\
\hline \multicolumn{6}{|c|}{ OPERADORA } & & & & & & & \\
\hline \multicolumn{6}{|c|}{ HOTÉIS } & & & & & & & \\
\hline
\end{tabular}

Fonte: Gráfico elaborado pelos autores (2015).

O Gráfico 3 constata que todos os entrevistados consideraram o serviço de academia no próprio hotel mais interessante do que a proximidade de um clube. Isto provavelmente se devendo à percepção dos pesquisados de que hóspedes do segmento de negócios prezam economia de tempo e praticidade.

GRÁFICO 4 - PREFERÊNCIA DOS HOTÉIS E OPERADORA EM RELAÇÃO AO SERVIÇO DE TV A CABO X INDICAÇÃO DE CINEMAS NAS PROXIMIDADES DO MEIO DE HOSPEDAGEM

\begin{tabular}{|c|c|c|c|c|c|c|c|c|c|c|c|c|}
\hline \multicolumn{6}{|c|}{ TV a cabo, sala de leitura etc. } & & \multicolumn{6}{|c|}{ Proximidade de cinemas etc. } \\
\hline 3,0 & 2,5 & 2,0 & 1,5 & 1,0 & 0,5 & 0,0 & 0,5 & 1,0 & 1,5 & 2,0 & 2,5 & 3,0 \\
\hline \multicolumn{6}{|c|}{ OPERADORA } & & & & & & & \\
\hline & & & & & IOTÉIS & & & & & & & \\
\hline
\end{tabular}

Fonte: Gráfico elaborado pelos autores (2015).

Ao verificar os dados dispostos no Gráfico 4, os entrevistados demonstraram preferência na oferta de serviço de TV a cabo aos hóspedes, em comparação à indicação de cinemas nas proximidades do meio de hospedagem. Revela-se, porém, que os 
gestores pesquisados deram pouca importância a esse serviço, e talvez isto tenha acontecido devido à percepção de que um turista de negócios possui pouco tempo livre, e que a televisão funciona como um passatempo apenas nos momentos de descanso e reclusão do hóspede em seus aposentos.

GRÁFICO 5 - PREFERÊNCIA DOS HOTÉIS E OPERADORA EM RELAÇÃO AO SERVIÇO DE SALA DE MASSAGEM E SAUNA X PROXIMIDADE A SPAS

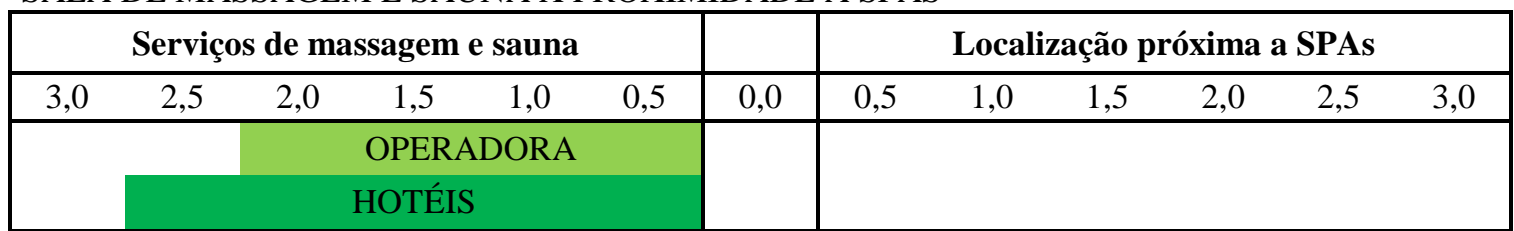

Fonte: Gráfico elaborado pelos autores (2015).

O Gráfico 5 mostra a preferência dos pesquisados pela disponibilização de sala de massagem e sauna aos hóspedes. Mais uma vez, para os gestores dos hotéis esse é um serviço de maior relevância, quando comparado à opinião da representante da Alatur. Vale lembrar que um serviço de massagem e sauna pode ser cobrado - o que representaria uma fonte adicional de vendas ao empreendimento. Uma análise de web sites de hotéis na cidade de São Paulo permitiu verificar que a incorporação de tal serviço à estrutura de um empreendimento tende a agregar valor ao portfólio de serviços oferecido por essa empresa e, de modo geral, é apresentado como um importante diferencial.

GRÁFICO 6 - PREFERÊNCIA DOS HOTÉIS E OPERADORA EM RELAÇÃO À LOCALIZAÇÃO DE BICICLETÁRIOS PRÓXIMOS AO MEIO DE HOSPEDAGEM X SERVIÇO DE LOCAÇÃO DE BICICLETAS

\begin{tabular}{|c|c|c|c|c|c|c|c|c|c|c|c|c|}
\hline \multicolumn{6}{|c|}{ Localização próxima a bicicletários } & & \multicolumn{6}{|c|}{ Serviço de locação de bicicletas } \\
\hline 3,0 & 2,5 & 2,0 & 1,5 & 1,0 & 0,5 & 0,0 & 0,5 & 1,0 & 1,5 & 2,0 & 2,5 & 3,0 \\
\hline & & & & & & & \multicolumn{6}{|c|}{ OPERADORA } \\
\hline & & & & & & & \multicolumn{4}{|c|}{ HOTÉIS } & & \\
\hline
\end{tabular}

Fonte: Gráfico elaborado pelos autores (2015).

Em relação à questão apresentada, todos os entrevistados optaram pela segunda alternativa - oferta de um serviço de aluguel de bicicletas aos hóspedes. Como forma de locomoção pela cidade, a ideia apresenta pontos positivos - contudo, os players do setor precisam considerar que custos estão associados à implantação de um serviço dessa natureza (ainda pouco comum entre as marcas presentes na cidade de São Paulo). 
Outro ponto a considerar é a viabilidade de tal investimento, pois embora São Paulo conte com equipamentos para lazer adequados, sua área de ciclovias ainda é reduzida: com "120,4 km de Ciclo Faixa de Lazer, mais os $30 \mathrm{~km}$ da Ciclovia da Marginal Pinheiros, que interliga o trecho sul/oeste ao trecho Guarapiranga" (CICLO FAIXA CIDADE DE SÃO PAULO, 2013).

GRÁFICO 7 - PREFERÊNCIA DOS HOTÉIS E OPERADORA EM RELAÇÃO À DISPONIBILIDADE DE SALA DE REUNIÕES NO MEIO DE HOSPEDAGEM X PROXIMIDADE DE CENTRO DE CONVENÇÕES

\begin{tabular}{|c|c|c|c|c|c|c|c|c|c|c|c|c|}
\hline \multicolumn{6}{|c|}{ Disponibilidade de salas para reuniões } & & \multicolumn{6}{|c|}{ Proximidade de Centro de Convenções } \\
\hline 3,0 & 2,5 & 2,0 & 1,5 & 1,0 & 0,5 & 0,0 & 0,5 & 1,0 & 1,5 & 2,0 & 2,5 & 3,0 \\
\hline \multicolumn{6}{|c|}{ OPERADORA } & & & & & & & \\
\hline \multicolumn{6}{|c|}{ HOTÉIS } & & & & & & & \\
\hline
\end{tabular}

Fonte: Gráfico elaborado pelos autores (2015).

O Gráfico 7 mostra que todos os entrevistados concederam maior importância à possibilidade de locação de salas de reuniões no próprio meio de hospedagem. Surpreendentemente, no entanto, essa opção foi considerada mais relevante pela representante da operadora do que pelos gestores dos hotéis. Isto talvez se deva ao fato de que os hotéis pesquisados se posicionavam proximamente localizados a um polo de teatros e espaços para convenções na região Sul da cidade e que, provavelmente, os hóspedes desses empreendimentos os escolhessem exatamente por tal motivo.

GRÁFICO 8 - PREFERÊNCIA DOS HOTÉIS E OPERADORA EM RELAÇÃO AO SERVIÇO DE TRANSFER 24 HORAS X PROXIMIDADE DE PONTOS DE TÁXI

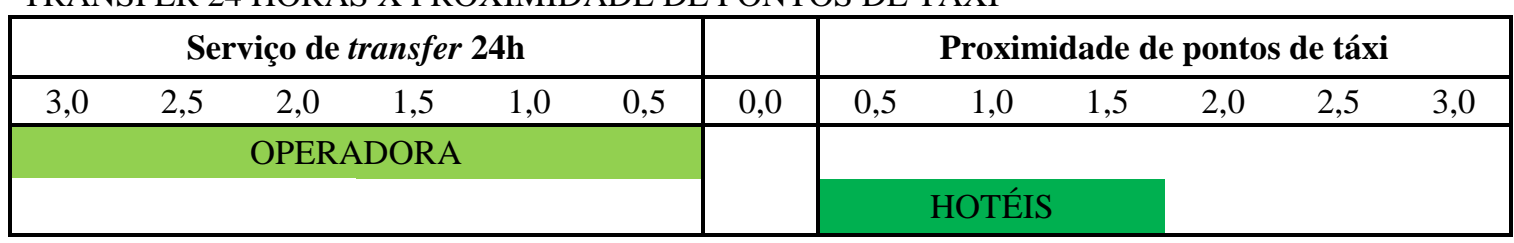

Fonte: Gráfico elaborado pelos autores (2015).

A análise do Gráfico 8 permite observar que os representantes das empresas pesquisadas demonstraram opiniões contrárias: enquanto a representante da operadora de viagens entendeu que a disponibilização de um serviço de transfer 24 horas fosse mais relevante para o hóspede, por representar uma facilidade ao cliente, os gestores dos hotéis pareceram acreditar que a proximidade de pontos de táxi era mais interessante para o hóspede. Também é possível inferir que o serviço de transfer, por representar um 
custo adicional, pode caracterizar-se como uma opção menos inteligente - sob o ponto de vista econômico - do que a "terceirização" do serviço por meio de táxis ou frotas particulares.

GRÁFICO 9 - PREFERÊNCIA DOS HOTÉIS E OPERADORA EM RELAÇÃO AO SERVIÇO DE EARLY CHECK-IN E LATE CHECK-OUT CORTESIA X SERVIÇO DE GUARDA-BAGAGEM NO MALEIRO

\begin{tabular}{|c|c|c|c|c|c|c|c|c|c|c|c|c|}
\hline \multicolumn{6}{|c|}{ Early check-in e late check-out } & & \multicolumn{6}{|c|}{ Guarda-bagagem cortesia } \\
\hline 3,0 & 2,5 & 2,0 & 1,5 & 1,0 & 0,5 & 0,0 & 0,5 & 1,0 & 1,5 & 2,0 & 2,5 & 3,0 \\
\hline & & & & & & OPER. & & & & & & \\
\hline & & & & OTÉ & & & & & & & & \\
\hline
\end{tabular}

Fonte: Gráfico elaborado pelos autores (2015).

É visível, pela análise do Gráfico 9, que essa questão é controversa mesmo entre os gestores dos hotéis - que pareceram não ter uma opinião formada a respeito do assunto. Os serviços de early check-in e late check-out envolvem aspectos operacionais do empreendimento, como a disponibilidade de unidades habitacionais, e sua adoção ou não podem impactar fortemente a percepção de satisfação do cliente.

Por vezes, verifica-se a cobrança pelos serviços de early check-in ou late checkout e, para tanto, os gestores devem levar em consideração o tempo necessário aos colaboradores da área de governança para preparar ou limpar as unidades habitacionais.

GRÁFICO 10 - PREFERÊNCIA DOS HOTÉIS E OPERADORA EM RELAÇÃO A DESCONTOS NAS DIÁRIAS NOS FINAIS DE SEMANA X DESCONTOS NAS DIÁRIAS DURANTE A SEMANA

\begin{tabular}{|c|c|c|c|c|c|c|c|c|c|c|c|c|}
\hline \multicolumn{6}{|c|}{$\begin{array}{l}\text { Desconto para hóspedes nos finais de } \\
\text { semana }\end{array}$} & & \multicolumn{6}{|c|}{$\begin{array}{c}\text { Tarifas reduzidas para empresas durante a } \\
\text { semana }\end{array}$} \\
\hline 3,0 & 2,5 & 2,0 & 1,5 & $\overline{1,0}$ & 0,5 & 0,0 & 0,5 & $\overline{1,0}$ & 1,5 & 2,0 & 2,5 & 3,0 \\
\hline & & & & & & & \multicolumn{6}{|c|}{ OPERADORA } \\
\hline & & & & & & & & $\mathrm{HO}^{-}$ & & & & \\
\hline
\end{tabular}

Fonte: Gráfico elaborado pelos autores (2015).

No que se refere aos dados expostos no Gráfico 10, verifica-se que os entrevistados demonstraram preferência por conceder descontos nas diárias no decorrer da semana, o que atenderia a uma demanda dos clientes corporativos. Para Estruquel (2013, p. 1) revela que a maior parte "dos hotéis são de investidores e existe muita pressão por resultados, baixa rentabilidade pode significar atrito dos investidores insatisfeitos com a administradora". Nesse contexto, um elemento de fidelização dos 
hóspedes é a paridade tarifária que engloba questões relacionadas a facilidade em adquirir e saldar as diárias do hotel.

GRÁFICO 11 - PREFERÊNCIA DOS HOTÉIS E OPERADORA EM RELAÇÃO A CAFÉ DA MANHÃ CORTESIA X PROXIMIDADE A PADARIAS E CASAS DE CHÁ

\begin{tabular}{|c|c|c|c|c|c|c|c|c|c|c|c|c|}
\hline \multicolumn{6}{|c|}{ Café da manhã } & & \multicolumn{6}{|c|}{ Proximidade a padarias etc. } \\
\hline 3,0 & 2,5 & 2,0 & 1,5 & 1,0 & 0,5 & 0,0 & 0,5 & 1,0 & 1,5 & 2,0 & 2,5 & 3,0 \\
\hline \multicolumn{6}{|c|}{ OPERADORA } & & & & & & & \\
\hline \multicolumn{6}{|c|}{ HOTÉIS } & & & & & & & \\
\hline
\end{tabular}

Fonte: Gráfico elaborado pelos autores (2015).

Pode-se observar, pelo Gráfico 11, que todos os entrevistados entenderam ser mais conveniente que o próprio meio de hospedagem oferecesse o café da manhã aos hóspedes. De acordo com Guerra (2001) é comum no Brasil que os meios de hospedagem não cobrem por esse serviço; verifica-se, no entanto, que a crescente globalização dos mercados e a entrada de novos players no cenário de turismo brasileiro vêm mudando esta realidade. Considera-se também que esta realidade é divergente em outros países, como por exemplo, nos Estados Unidos (GUERRA, 2001).

GRÁFICO 12 - PREFERÊNCIA DOS HOTÉIS E OPERADORA EM RELAÇÃO AO SERVIÇO DE ESTACIONAMENTO CORTESIA $X$ LOCALIZAÇÃO PRÓXIMA A PONTOS DE TÁXI E TERMINAIS DE ONNIBUS

\begin{tabular}{|ccc|c|ccccc|}
\hline \multicolumn{3}{|c|}{ Estacionamento } & & \multicolumn{5}{c|}{ Proximidade a pontos de táxi etc. } \\
\hline $3,0 \quad 2,5 \quad 2,0 \quad 1,5 \quad 1,0$ & 0,5 & 0,0 & 0,5 & 1,0 & 1,5 & 2,0 & 2,5 & 3,0 \\
\hline \multicolumn{3}{c|}{ OPERADORA } & & & & & & \\
\hline \\
HOTÉIS & & & & & & \\
\hline
\end{tabular}

Fonte: Gráfico elaborado pelos autores (2015).

Ao examinar o Gráfico 12, verifica-se que os gestores de hotéis e a representante da operadora pesquisada manifestaram preferir que o meio de hospedagem oferecesse o serviço de estacionamento cortesia a seus clientes.

Esta não parece ser uma tarefa simples em uma cidade como São Paulo: nos dias atuais, faltam vagas de estacionamento em algumas regiões da metrópole e uma solução seria a cobrança ou a terceirização do serviço, o que - se por um lado representaria uma fonte extra de receita para a empresa - por outro deixaria de ser uma cortesia e, portanto, menos interessante ao hóspede. 
GRÁFICO 13 - PREFERÊNCIA DOS HOTÉIS E OPERADORA EM RELAÇÃO AO SERVIÇO DE LOCAÇÃO DE VEÍCULOS X PROXIMIDADE A PONTOS DE TÁXI E TERMINAIS DE ÔNIBUS

\begin{tabular}{|c|c|c|c|c|c|c|c|c|c|c|c|c|}
\hline \multicolumn{6}{|c|}{ Serviço de locação de veículos } & & \multicolumn{6}{|c|}{ Proximidade a pontos de táxi etc. } \\
\hline 3,0 & 2,5 & 2,0 & 1,5 & 1,0 & 0,5 & 0,0 & 0,5 & 1,0 & 1,5 & 2,0 & 2,5 & 3,0 \\
\hline \multicolumn{6}{|c|}{ OPERADORA } & & & & & & & \\
\hline
\end{tabular}

Fonte: Gráfico elaborado pelos autores (2015).

Oferecer um serviço para locação de veículos parece ser uma alternativa bem vinda tanto aos hotéis quanto à operadora. Para a operadora, inclusive, este serviço foi considerado extremamente importante, ao passo que para os hotéis ele teria um peso mais equilibrado. Entende-se, a partir desse resultado, que o fato de um hotel dispor do serviço de locação de veículos em suas próprias instalações pode agregar valor ao seu portfólio de serviços, porém o fato de esse hotel estar instalado nas proximidades de pontos de táxi ou de terminais de ônibus também se constitui em uma facilidade para os hóspedes.

GRÁFICO 14 - PREFERÊNCIA DOS HOTÉIS E OPERADORA EM RELAÇÃO AO SERVIÇO DE CONCIÉRGE X PROXIMIDADE A TEATROS E CINEMAS

\begin{tabular}{|c|c|c|c|c|c|c|c|c|c|c|c|c|}
\hline \multicolumn{6}{|c|}{ Serviço de conciérge } & & \multicolumn{6}{|c|}{ Proximidade a teatros e cinemas } \\
\hline 3,0 & 2,5 & 2,0 & 1,5 & 1,0 & 0,5 & 0,0 & 0,5 & 1,0 & 1,5 & 2,0 & 2,5 & 3,0 \\
\hline \multicolumn{6}{|c|}{ OPERADORA } & & & & & & & \\
\hline & & & & & & HOTÉ & & & & & & \\
\hline
\end{tabular}

Fonte: Gráfico elaborado pelos autores (2015).

Da mesma forma que no Gráfico 9, neste Gráfico 14 os gestores dos hotéis pesquisados denotaram dubiedade de resposta. Segundo a Les Clefs d’Or Brésil Associação Brasileira de Conciérges (2013), este profissional, quando no uso de suas atribuições, "informa, aconselha, sugere, acompanha, assiste aos hóspedes de um hotel de todas as formas possíveis".

Esta assistência tende a ser descrita ou é disposta na forma de "serviços hoteleiros, facilidades recreacionais, indicações e reservas de restaurantes, envio e guarda de bagagem, aluguel de transportes, indicação de tours e etc.” (LES CLEFS D OR BRÉSIL - ASSOCIAÇÃO BRASILEIRA DE CONCIÉRGES, 2013). Talvez o fato de que o conciérge seja considerado, por suas características, um serviço requintado e de luxo - e, por isso, nem sempre desfrutado por hóspedes que buscam praticidade e 
repouso - tenha contribuído para o resultado desta questão por parte dos gestores hoteleiros.

GRÁFICO 15 - PREFERÊNCIA DOS HOTÉIS E OPERADORA EM RELAÇÃO AO SERVIÇO DE LAVANDERIA 24 HORAS X PROXIMIDADE A LAVANDERIAS

\begin{tabular}{|lcll|l|lllll|}
\hline \multicolumn{3}{|c|}{ Lavanderia 24h } & & \multicolumn{5}{c|}{ Proximidade a lavanderias } \\
\hline $3,0 \quad 2,5 \quad 2,0 \quad 1,5$ & 1,0 & 0,5 & 0,0 & 0,5 & 1,0 & 1,5 & 2,0 & 2,5 & 3,0 \\
\hline \multicolumn{3}{c|}{ OPERADORA } \\
HOTÉIS & & & & & & & & \\
\hline
\end{tabular}

Fonte: Gráfico elaborado pelos autores (2015).

Entre hotéis de médio e grande porte, disponibilizar o serviço de lavanderia é uma atividade comum e, pelo resultado apresentado no Gráfico 15, uma unanimidade quando considerada sua importância para o hóspede corporativo.

GRÁFICO 16 - PREFERÊNCIA DOS HOTÉIS E OPERADORA EM RELAÇÃO AO SERVIÇO DE COFRE 24 HORAS X PROXIMIDADE A BANCOS E CASAS LOTÉRICAS

\begin{tabular}{|c|c|c|c|c|c|c|c|c|c|c|c|c|}
\hline \multicolumn{6}{|c|}{ Cofre 24h } & & \multicolumn{6}{|c|}{ Proximidade a bancos e casas lotéricas } \\
\hline 3,0 & 2,5 & 2,0 & 1,5 & 1,0 & 0,5 & 0,0 & 0,5 & 1,0 & 1,5 & 2,0 & 2,5 & 3,0 \\
\hline \multicolumn{6}{|c|}{ OPERADORA } & & & & & & & \\
\hline \multicolumn{6}{|c|}{ HOTÉIS } & & & & & & & \\
\hline
\end{tabular}

Fonte: Gráfico elaborado pelos autores (2015).

Ao investigar os dados dispostos no Gráfico 16, verifica-se que todos os entrevistados compreenderam que, para o turista de negócios, a opção por um cofre 24 horas é a que melhor atende a suas necessidades. Isto se dá em função da segurança que o meio de hospedagem proporciona ao hóspede e que, por expansão, acaba sendo avaliada também sob a ótica de seus pertences.

GRÁFICO 17 - PREFERÊNCIA DOS HOTÉIS E OPERADORA EM RELAÇÃO AO SERVIÇO DE FRIGOBAR X INDICAÇÃO DE RESTAURANTES NAS PROXIMIDADES

\begin{tabular}{|c|c|c|c|c|c|c|c|c|c|c|c|c|}
\hline \multicolumn{6}{|c|}{ Frigobar } & & \multicolumn{6}{|c|}{ Proximidade a restaurantes } \\
\hline 3,0 & 2,5 & 2,0 & 1,5 & 1,0 & 0,5 & 0,0 & 0,5 & 1,0 & 1,5 & 2,0 & 2,5 & 3,0 \\
\hline \multicolumn{6}{|c|}{ OPERADORA } & & & & & & & \\
\hline \multicolumn{6}{|c|}{ HOTÉIS } & & & & & & & \\
\hline
\end{tabular}

Fonte: Gráfico elaborado pelos autores (2015).

Por meio de análise do Gráfico 17, verifica-se que a presença de um frigobar na unidade habitacional foi entendida, pelos gestores dos hotéis e pela representante da 
operadora pesquisados, como um serviço interessante para o hóspede. Para os hotéis, no entanto, o serviço é considerado de relevância expressiva, o que talvez indique que, além da comodidade ao turista, o frigobar também funcione como uma fonte adicional de receitas para o hotel.

GRÁFICO 18 - PREFERÊNCIA DOS HOTÉIS E OPERADORA EM RELAÇÃO AO SERVIÇO DE PASSADORIA 24 HORAS X EMPRÉSTIMO DE FERRO DE PASSAR

\begin{tabular}{|c|c|c|c|c|c|c|c|c|c|c|c|c|}
\hline \multicolumn{6}{|c|}{ Passadoria 24h } & & \multicolumn{6}{|c|}{ Empréstimo de ferro de passar } \\
\hline 3,0 & 2,5 & 2,0 & 1,5 & 1,0 & 0,5 & 0,0 & 0,5 & 1,0 & 1,5 & 2,0 & 2,5 & 3,0 \\
\hline \multicolumn{6}{|c|}{ OPERADORA } & & & & & & & \\
\hline \multicolumn{6}{|c|}{ HOTÉIS } & & & & & & & \\
\hline
\end{tabular}

Fonte: Gráfico elaborado pelos autores (2015).

Entre o empréstimo de ferro elétrico e a oferta de um serviço de passadoria, os entrevistados optaram por este serviço. É preciso considerar que, sob a ótica da praticidade para o hóspede, ambas as opções podem ser valiosas; sob a ótica de um empreendimento voltado ao lucro, a presença de um serviço 24 horas (e a possibilidade de cobrar por isso) adquire maior interesse enquanto negócio.

\section{CONSIDERAÇÕES FINAIS}

Por meio deste estudo buscou-se analisar a prestação de serviços nos meios de hospedagem da cidade de São Paulo. Ao seguir o cenário de transformações existente no ambiente global e ao obedecer a lógica da globalização, pretendeu-se compreender de que modo as necessidades dos consumidores podem ser satisfeitas a partir das imposições estatais dos regulamentos e normas do setor.

No caso deste artigo, usou-se como modelo referencial a Publicação Placar da Hotelaria (SENAC SÃO PAULO, 2013) e o Anuário Estatístico do Turismo (BRASIL, 2013), de forma demonstrar respectivamente segmentação do mercado por tipos e classes e também a expressiva ampliação do mercado turístico internacional para o Brasil.

De forma a demonstrar as tendências globais e a relação complexa entre hotéis e operadores de turismo, tomou-se por base estudos da área que demonstram as 
tendências do setor e, por meio da pesquisa aplicada, foi possível entender que os gestores disponibilizam, em seus empreendimentos, serviços que sejam - ao mesmo tempo - fonte de receita e índice de valor ao portfólio da empresa. Enquanto a representante da operadora de viagens deu maior ênfase a serviços que tendem a agradar e satisfazer sem necessariamente representar ônus para os hóspedes, os gestores dos hotéis indicaram, em suas respostas, uma preocupação adicional com a rentabilidade de uma prestação de serviço. Espera-se, por meio desta pesquisa, contribuir no âmbito acadêmico com os estudos voltados e focados ao desenvolvimento do setor de turismo e hospitalidade na cidade de São Paulo e, particularmente, no Brasil.

\section{REFERÊNCIAS}

ALATUR JTB. A ALATUR JTB. Disponível em: <http://www.alaturjtb.com/alaturjtb>. Acesso em: 20/07/2015.

ALATUR JTB. Alcance Global. Disponível em: <http://www.alaturjtb.com/alcanceglobal>. Acesso em: 20/07/15.

BRASIL. MINISTÉRIO DO TURISMO. Anuário Estatístico do Turismo 2013. Disponível em: <www.dadosefatos.turismo.gov.br/dadosefatos/anuario/>. Acesso em: 30/07/2013.

BRASIL. MINISTÉRIO DO TURISMO. Gabinete do Ministro. Portaria $\mathrm{n}^{\circ} 100$, de 16 de junho de 2011. 2015. <On line> Disponível em: $<$ http://www.classificacao.turismo.gov.br/MTUR-classificacao/mtursite/downloads/portaria100_2011mtur.pdf>. Acesso em: 20/07/15.

BUHALIS, D. Relationships in the Distribution Channel of Tourism: Conflicts Between Hoteliers and Tour Operators in the Mediterranean Region. International Journal of Hospitality \& Tourism Administration, v. 1, n. 1, 2000, p. 113-139.

CASTELLS, M. Materials for an exploratory theory of the network society. British Journal of Sociology, v. 51, n. 1, January/March, p. 5-24. 2000.

CICLO FAIXA CIDADE DE SÃO PAULO. Disponível em: <www.ciclofaixa.com.br/o-projeto/>. Acesso em: 05/08/2013.

CONVENTION VISITORS BUREAU. Disponível em: $<$ http://visitesaopaulo.com/dados-da-cidade.asp>. Acesso em: 23/07/15. 
DELOITTE CONSULTING SERVICES. Resetting Horizons - Global Human Capital Trens 2013. Disponível em: <http://www2.deloitte.com/sa/en/pages/humancapital/articles/global-human-capital-trends-2013.html>. Acesso em 09/07/2013.

ESTRUQUEL, W. A escolha correta de canais de distribuição e vendas nos hotéis. Disponível em: <www.revistahoteis.com.br/materias/view/9602>. Acesso em: 10/07/2013.

GUERRA, G. R. Preço de Transferência: Tratamento Contábil do Café da Manhã pela Hotelaria Paulistana. Turismo em Análise. São Paulo, v. 12, n. 1, maio, 2001.

LOPES, A. S.; MOLA, J. L. Serviços essenciais para o turista de negócios sob a ótica dos operadores de turismo. Anais do Seminário da Associação Nacional de Pesquisa e Pós-graduação em Turismo, 2013.

LES CLEFS D OR BRÉSIL - ASSOCIAÇÃO BRASILEIRA DE CONCIÉRGES. Disponível em: <d701101147.tecla143.tecla.com.br/site/pt-br/concierge/>. Acesso em: 05/08/2013.

NASCIMENTO, R. C.; LOPES, A. S. Análise do referencial teórico sobre turismo de negócios no Banco de Teses da CAPES. Anais do Seminário da Associação Nacional de Pesquisa e Pós-graduação em Turismo, 2012.

OTTO, G. 4 tendências da hotelaria para 2013. Disponível em: <hoteliernews.com.br/2013/04/4-tendencias-da-hotelaria-para-2013/>. Acesso em: 09/07/2013.

PANOSSO NETTO, A; CALCIOLARI, G. F. de M. Quantos são os livros teóricos de turismo publicados no Brasil? Uma análise da produção bibliográfica nacional (19902010). Turismo em análise, v. 21, n. 3, dezembro, 2010.

TRAVEL + LEISURE. Most important Travel Trends in 2014. Disponível em: < http://www.travelandleisure.com/slideshows/most-important-travel-trends-2014>, Acesso em: 09/07/2013.

SANTOS, J. A. dos; SANTOS, G. E. de O. Uso de smartphones em viagens de turismo: análise do comportamento do mercado paulistano. Turismo \& Sociedade, v. 7, n. 4, outubro, 2014.

SENAC SÃO PAULO. Placar da Hotelaria. Disponível em: <www.sp.senac.br/placardahotelaria>. Acesso em: 20/07/2013.

Recebido em: 25-06-2014.

Aprovado em: 25-07-2014. 